\title{
Linguistic Repertoire and Sociolinguistic Contexts for Acquisition of Burushaski and Other Languages by Burushos of Kashmir
}

\author{
Sabba Mushtaq \\ University of Kashmir, Dept of Linguistics, Hazratbal, Srinagar, Kashmir, India
}

\begin{abstract}
Burushaski, Pashto, Dardi and Gojri are regarded as minority languages as their speakers are demographically lower in number in Jammu and Kashmir and the natives of these languages use Kashmiri or Urdu in formal domains. Burushaski, also known by the names of Boorishki, Brugaski, Kanjut, Werchikwar and Mishaski is a language isolate spoken by some 87,000 Burusho people in Hunza, Nagar and Yasin area of Northern Pakistan. Linguistically, Burushaski has been termed as a language isolate because it does not characterize the genetic relationship with the surrounding language families like Indic, Sino-Tibetan, Dardic etc. Ethnologue (2005) mentions Burushaski speakers in India but does not provide about the specific information about the number of speakers and their exact location. According to the members of this speech community 300-350 Burushos live in Srinagar. Burushaski speakers of Jammu and Kashmir are settled in and around a small locality by the foothills of Hariparbat in Srinagar, the capital of Jammu and Kashmir. The locality is known as Mohalla Azur Khan, named after Raja Azur Khan. Jammu and Kashmir Burushos are also reported to live in Tral (Pulwama), Batamaloo, and Dandusa in Bemina area of Srinagar. The present paper aims to study the sociolinguistic context for acquisition of Burushaski and other languages known to Burushos of Kashmir.
\end{abstract}

Keywords: Sociolinguistic Context, Linguistic Repertoire, Language Isolate, Burushaski

\section{Introduction}

Burushos form a minority speech community of J \& K. The dominant language which is spoken within this community is their mother tongue i.e. Burushaski. Burushaski is acquired mainly in the home domain. The other languages known by this community are Balti, Kashmiri, Urdu, and English which are acquired from various sociolinguistic contexts like school, environment, social interactions, mass media etc. All the five languages i.e. Burushaski, Balti, Kashmiri, Urdu and English form the linguistic repertoire of Burushos. The acquisition of Burushaski, Balti, Kashmiri, Urdu and English are represented in fig 1 to 5.The sociolinguistic contexts like Home, Environment and School have been taken into consideration for present study. In these figures $\mathrm{H}$ stands for home, E for Environment (other than Home, and school) and S for School.

\section{Methodology}

The present study is based on the data collected from 76 language respondents during an intensive field work. The language respondents have been divided in social variables viz a) Age and b) Gender. Education although may be a significant social variable in some other studies but in the present study it has been observed that Burushos are educated across age and gender. The pilot survey has given the idea that education is not a relevant sociolinguistic variable to measure the issues related to the present study. The respondents were taken from Mohalla Azur Khan, Kathi Darwaza, Srinagar. All the respondents were the native speakers of Burushaski.

The questionnaire acted as a tool for eliciting the sociolinguistic data from the Burushos speech community. It consists of two sections. Each section has its own focus.
Section I focused on discovering the background information of the subjects such as age, gender, education level and occupation.

Section II is aimed to determine the sociolinguistic contexts in which Burushaski, Balti, Kashmiri, Urdu and English are learned. The respondents were asked where they acquired Burushaski, Balti, Kashmiri, Urdu, and English. The scoring was done as follows:

Table 1: Scale for Sociolinguistic context for acquisition

\begin{tabular}{|l|l|}
\hline In Home & 1 \\
\hline At school & 2 \\
\hline Environment & 3 \\
\hline
\end{tabular}

Higher score indicates that the informant has acquired the language from environment and the lower score indicates that the respondents have acquired the language in his home. The data was then codified, tabulated and quantified for every respondent

\section{Results}

The quantification of the data revealed following results

a) Burushaski: Sociolinguistic context for acquisition 


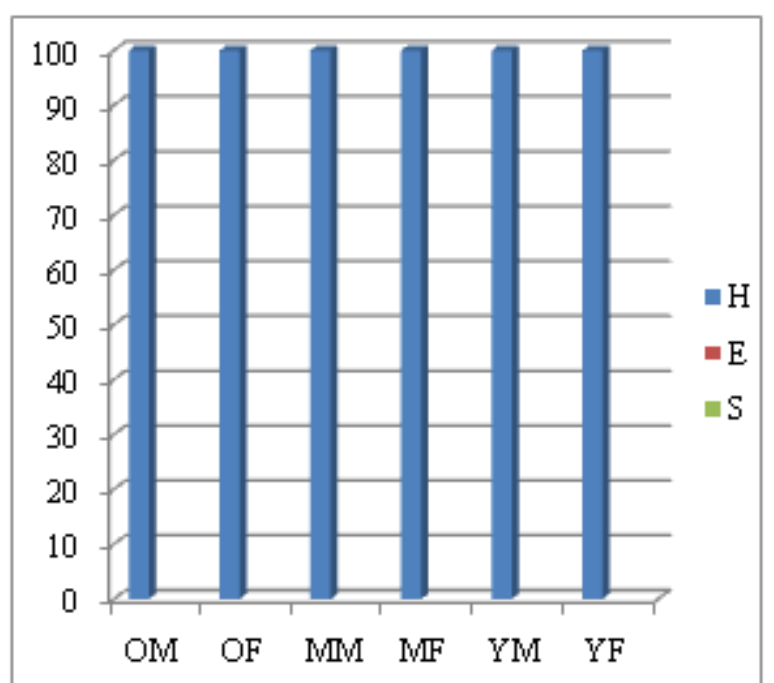

Figure 1: Sociolinguistic context for acquisition of Burushaski

The fig 1 shows that most of the respondents in all age groups have acquired Burushaski at home. Males and females irrespective of all age groups exclusively use Burushaski for communicative purposes within the home domain. Burushaski being their mother tongue occupies a high place in their community and is related to their identity. When a child is born in their community the first language they acquire is Burushaski which they learn in their home domain. In general it is clear that Burushaski is the language of home domain. It is evident from the above graph that Burushos are essentially attached to their mother tongue i.e. Burushaski. Hence, Burushaski occupies a central position of their linguistic repertoire.

b) Balti: Sociolinguistic context for acquisition

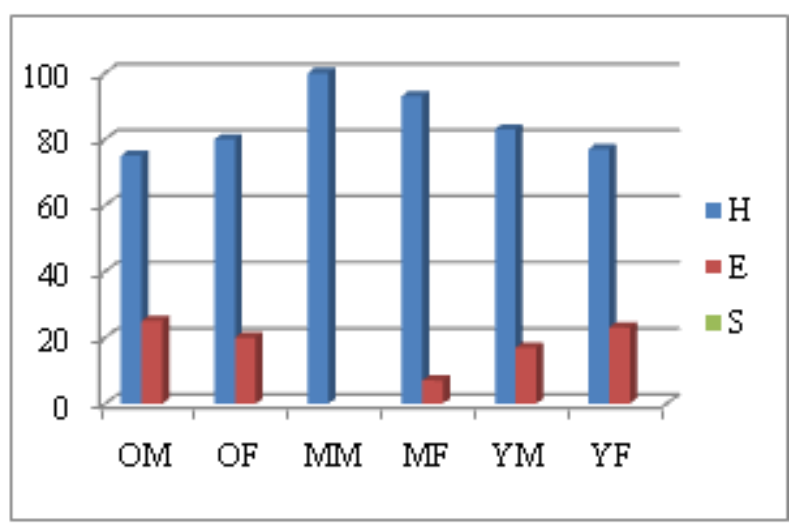

Figure 2: Sociolinguistic context for acquisition of Balti

The fig 2 shows that Balti is acquired at home. Middle aged males claim that they have acquired Balti from the environment because of their intimate social contacts with Balti speakers. The following factors appear to be responsible for shaping Balti language as one of the languages in the home domain:

1) Intermarriage with Balti speaking community.

2) Engage servants of Balti community for domestic help. c) Kashmiri: Sociolinguistic context for acquisition

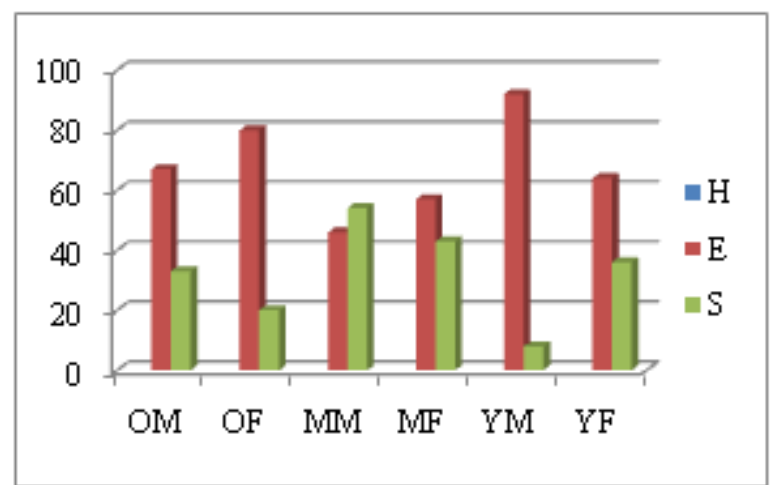

Figure 3: Sociolinguistic context for acquisition of Kashmiri

Fig 3 shows the contexts in which respondents have claimed to have acquired Kashmiri. All the age groups have acquired Kashmiri from the environment and school. The old aged and middle aged groups claim to have learnt Kashmiri either from informal conversation with teacher or fellow being in Kashmiri during schooling. In case of young aged groups some respondents claim to have learnt Kashmiri in schools. In the beginning of the present decade the language policy adopted by the government has influenced the acquisition of Kashmiri language among Burushos. Thus, according to the present study the section of age group (14-25 years) claim to have learnt Kashmiri language along with other languages of school curriculum.

d) Urdu: Sociolinguistic context for acquisition

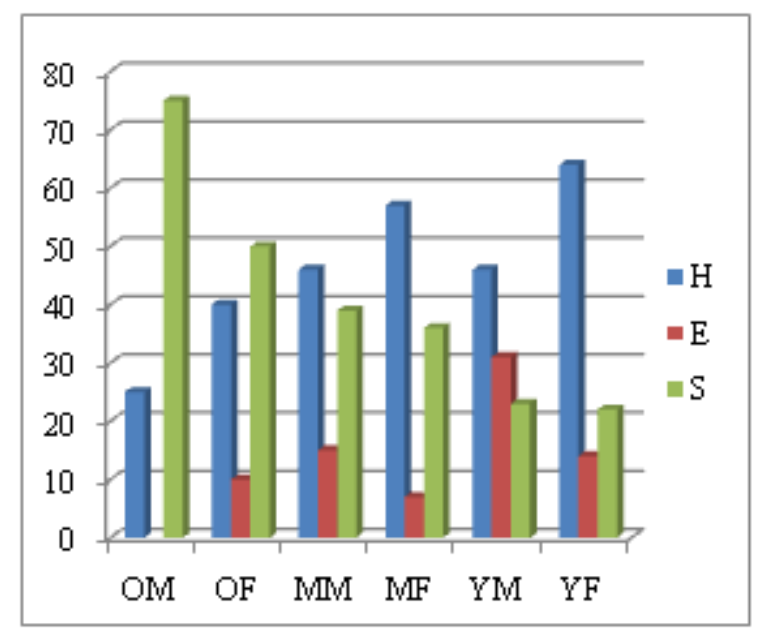

Figure 4: Sociolinguistic context for acquisition of Urdu

The fig 4 shows that Urdu has been acquired from all the three domains. The present study shows that importance of school as context of the acquisition of Urdu decreases from older generation to younger generation and the importance of home domain increases from older to younger generation. The increase shown in the use of English by younger generation can be attributed to the fact that after Burushaski, Urdu occupies a predominant place in the home domain. Except for old aged males all the age groups claim that they have acquired Urdu from the environment. 
e) English: Sociolinguistic context for acquisition

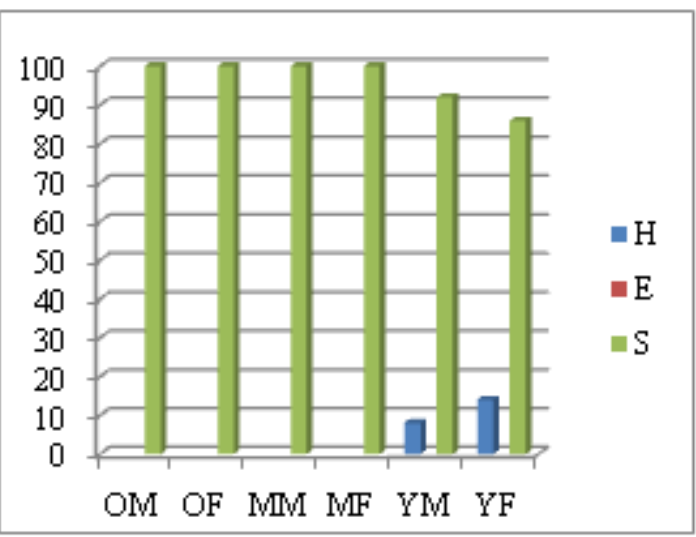

Figure 5: Sociolinguistic context for acquisition of English

Fig 5 shows that majority of the groups learn English at school in form of curriculum in school education.Apparently $20 \%$ of younger generation learn English in home domain as well. There has been a trend to use English at home because of the prestige tag and high status attached to the language. Further this language can provide better career opportunities in life.

\section{Conclusion}

Linguistically Burushos are acquainted with languages like Burushaski, Balti, Kashmiri, Urdu and English.Burushos acquisition of these languages and their functions in various social domains vary. The sociolinguistic Parameters evolved in the speech community play a considerable role in acquisition and use of these languages. Burushaski language is mostly acquired in the home domain.Urdu is usually acquired at home as well as the school. Urdu is ascribed a more positive value than Kashmiri by the Burushos as Urdu is considered a language of education and literacy. Kashmiri, is acquired from surrounding environment which includes neighborhood, school etc. The Kashmiri language to be the part of their linguistic repertoire is obligatory and not optional. English is acquired from educational institutions.

\section{References}

[1] Colin Baker, Attitudes and Language, Multilingual Matters Ltd, Philadelphia, 1992.

[2] C. Butler, Statistics in Linguistics, Basil Blackwell,New York,1985.

[3] Dick Grunes, Burushaski: An Extraordinary language in the Korakoram Mountains, Joseph Biddulph publishers, UK, 1998.

[4] G.A Grierson, Linguistic Survey of India, Volume X, Munshi Ram Manoharlal Publishers, Delhi, 1889.

[5] H.M. Batibo, Language decline and death in Africa:Causes, Consequences and Challenges, Multilingual Matters Ltd., Toronto,2005.

[6] Michael C. Shapiro, \& Harold Schiffman, Language and Society in South Asia, Motilal Banarasidass Publishers Pvt.Ltd, New Delhi,1981.

[7] R. Fasold, The Sociolinguistics of Society, Basil Blackwell, New York, 1989.
[8] Sadaf Munshi, Jammu and Kashmir Burushaski: Language, Language Contact and Change, Ph.D Dissertation, University of Texas, Austin, 2006.

[9] Shahnaz Hunzia, Burushaski-Urdu Dictionary, Burushaski Research Academy, Hunza, 2009. 